Алгебра и анализ

Том 20 (2008), № 3
St. Petersburg Math. J.

Vol. 20 (2009), No. 3, Pages 325-337 S 1061-0022(09)01050-4

Article electronically published on April 6, 2009

\title{
ON THE MAXIMUM PRINCIPLE FOR HARMONIC FUNCTIONS
}

\author{
A. VAGHARSHAKYAN
}

\begin{abstract}
Some generalizations of the maximum principle for harmonic functions
\end{abstract} are discussed.

\section{§1. INTRODUCTION}

It is well known that if $U(z),|z|<1$, is a harmonic function and for each boundary point $e^{i x}$ we have

$$
\limsup _{z \rightarrow e^{i x}} U(z) \leq 0,
$$

then $U(z) \leq 0$ everywhere on the unit disk $\{z ;|z|<1\}$. If we know a priori that $U(z),|z|<1$, is bounded, then by Fatou's theorem we need to check condition (1) at almost all boundary points only. Moreover, we can replace (1) with the weaker condition where the point $z$ goes to $e^{i x}$ nontangentially. By the Phragmén-Lindelöf principle, if

$$
\max _{x}\left|U\left(r e^{i x}\right)\right|=o\left(\frac{1}{1-r}\right),
$$

then we can omit finitely many boundary points. There are many results in this direction, where various conditions are imposed on harmonic functions and the corresponding exceptional sets are characterized.

In this paper we discuss the problem of relaxing condition (1) at each boundary point provided an additional condition is imposed on the growth of the harmonic function in question. We also discuss the problem of omitting some boundary points.

\section{§2. Auxiliary Results}

In this section, for the reader's convenience, we collect some well-known results and definitions.

More information about the following quantity, related to the Hausdorff measure, can be found in [2].

Definition 2.1. Let $0<\alpha \leq 1$. Suppose a subset $E \subset\{z ;|z|=1\}$ is covered with a family of $\operatorname{arcs}\left\{S_{k}\right\}_{k=1}^{\infty}$, i.e.,

$$
E \subseteq \bigcup_{k=1}^{\infty} S_{k}
$$

Then we put

$$
M_{\alpha}(E)=\inf \left(\sum_{k=1}^{\infty}\left|S_{k}\right|^{\alpha}\right),
$$

2000 Mathematics Subject Classification. Primary 30C80, 31A15.

Key words and phrases. Harmonic functions, maximum principle.

(C)2009 American Mathematical Society 
where $|S|$ is the length of the arc $S$ and the infimum is taken over the family of all covers.

The following definition and theorem are borrowed from the book [3].

Definition 2.2. Suppose $1 \leq p<\infty$ and $0<\alpha<1$. A function $f(t), 0 \leq t \leq 2 \pi$, belongs to the Besov space $\Lambda_{\alpha}^{p}$ if

$$
\|f\|_{\Lambda_{\alpha}^{p}}^{p}=\int_{-\pi}^{\pi}|f(t)|^{p} d t+\int_{-\pi}^{\pi} \int_{-\pi}^{\pi} \frac{|f(t)-f(x)|^{p}}{|x-t|^{1+\alpha p}} d t d x<\infty .
$$

Theorem 2.1. A function $f(t), 0 \leq t \leq 2 \pi$, belongs to the space $\Lambda_{\alpha}^{p}$, where $0<\alpha<1$ and $1 \leq p<\infty$, if and only if

$$
\int_{0}^{1} \int_{-\pi}^{\pi}\left|\frac{\partial F\left(r e^{i t}\right)}{\partial r}\right|^{p} \frac{d r d t}{(1-r)^{1-p+\alpha p}}<\infty
$$

where $F(z)$ is the following harmonic function:

$$
F(z)=\frac{1}{2 \pi} \int_{-\pi}^{\pi} \frac{1-|z|^{2}}{\left|e^{i t}-z\right|^{2}} f(t) d t, \quad|z|<1 .
$$

The proof of the following classical result can be found in [1, p. 84].

Theorem 2.2. Let $C(x), x \geq 0$, be a monotone nondecreasing function with

$$
\int_{1}^{\infty} \frac{C(x)}{x^{2}} d x<\infty
$$

Then for an arbitrary positive number a there is a nonnegative function $f(x) \not \equiv 0$ that vanishes outside of the interval $(-a, a)$ and satisfies

$$
|\hat{f}(x)| \leq \exp (-C(|x|)), \quad x \in(-\infty, \infty),
$$

where $\hat{f}(x)$ is the Fourier transform of the function $f(x)$.

\section{§3. The CASE WHERE THE LENGTHS OF THE ARCS GO TO ZERO}

Theorem 3.1. Suppose $1 \leq p<\infty, 0<\alpha<\frac{1}{p}$. Let $E$ be a closed subset of the unit circle $T$, and let

$$
M_{1-\alpha p}(E)=0 .
$$

Let $l \in\left(\Lambda_{\alpha}^{p}\right)^{*}$ be a bounded linear functional such that $l(f) \geq 0$ for each nonnegative function $f(x) \in \Lambda_{\alpha}^{p}$ satisfying

$$
\operatorname{supp}(f) \subset T \backslash E \text {. }
$$

Then for each nonnegative function $f(x) \in \Lambda_{\alpha}^{p}$ we also have $l(f) \geq 0$.

Proof. Observe that it suffices to prove the inequality $l(f) \geq 0$ for all nonnegative and continuously differentiable functions $f(x)$.

For an arbitrary $\epsilon>0$, there are open $\operatorname{arcs} I_{n}, n=1,2, \ldots$, such that

$$
E \subset F=\bigcup_{n=1}^{\infty} I_{n}
$$

and

$$
\sum_{n=1}^{\infty}\left|I_{n}\right|^{1-\alpha p}<\epsilon
$$

We denote by $f_{0}(x)$ the following function:

and

$$
f_{0}(x)=f(x), \quad x \in T \backslash F,
$$

$$
f_{0}(x)=0, \quad x \in F
$$


By assumption, we have $l\left(f_{0}\right) \geq 0$. Next,

$$
\begin{aligned}
\left\|f-f_{0}\right\|_{\Lambda_{\alpha}^{p}}^{p} & =\int_{F}|f(x)|^{p} d x+\int_{F} \int_{F} \frac{|f(x)-f(t)|^{p}}{|x-t|^{1+\alpha p}} d x d t+2 \int_{F} \int_{T \backslash F} \frac{|f(x)|^{p}}{|x-t|^{1+\alpha p}} d x d t \\
& \leq M\left(|F|+\int_{F} \int_{F} \frac{|x-t|^{p}}{|x-t|^{1+\alpha p}} d x d t+2 \sum_{n=1}^{\infty} \int_{I_{n}} \int_{T \backslash F} \frac{1}{|x-t|^{1+\alpha p}} d x d t\right) \\
& \leq M\left(|F|+\int_{0}^{|F|} \int_{0}^{|F|} \frac{d x d t}{|x-t|^{1-p+\alpha p}}+\sum_{n=1}^{\infty}\left|I_{n}\right|^{1-\alpha p}\right) \leq M_{1} \epsilon
\end{aligned}
$$

where $M_{1}$ is independent of $\epsilon$.

Lemma 3.1. Suppose $U(z)$ and $V(z),|z|<1$, are harmonic functions that admit continuous extension up to the boundary of the unit disk. Then for each $0<\rho<1$ we have

$$
\begin{aligned}
\frac{1}{2 \pi} \int_{-\pi}^{\pi} U\left(\rho e^{i t}\right) V\left(e^{i t}\right) d t & =\frac{1}{2 \pi} \int_{-\pi}^{\pi} U\left(e^{i t}\right) V\left(\rho e^{i t}\right) d t \\
& =U(0) V(0)+\frac{1}{\pi} \int_{0}^{\sqrt{\rho}} \int_{-\pi}^{\pi} U\left(r e^{i t}\right) \frac{\partial V\left(r e^{i t}\right)}{\partial r} d r d t
\end{aligned}
$$

Proof. Let

and

$$
U\left(r e^{i t}\right)=\sum_{n=-\infty}^{\infty} u_{n} r^{|n|} e^{i n t}
$$

Then

$$
V\left(r e^{i t}\right)=\sum_{n=-\infty}^{\infty} v_{n} r^{|n|} e^{i n t}
$$

Consequently,

$$
\frac{1}{2 \pi} \int_{-\pi}^{\pi} U\left(r e^{i t}\right) \frac{\partial V\left(r e^{i t}\right)}{\partial r} d t=\sum_{n \neq 0} u_{n} v_{-n}|n| r^{2|n|-1}
$$

$$
\begin{aligned}
\frac{1}{2 \pi} \int_{0}^{\sqrt{\rho}} \int_{-\pi}^{\pi} U\left(r e^{i t}\right) \frac{\partial V\left(r e^{i t}\right)}{\partial r} d r d t & =\frac{1}{2} \sum_{n \neq 0} u_{n} v_{-n} \rho^{|n|} \\
& =\frac{1}{4 \pi} \int_{-\pi}^{\pi} U\left(\rho e^{i t}\right) V\left(e^{i t}\right) d t-\frac{U(0) V(0)}{2} .
\end{aligned}
$$

Lemma 3.2. Suppose $1 \leq p<\infty, 0<\alpha p<1$. Then any harmonic function $V(z)$, $|z|<1$, that satisfies the condition

for $p=1$ and the condition

$$
|V(z)| \leq \frac{M}{(1-|z|)^{\alpha}}, \quad|z|<1
$$

$$
\int_{0}^{1} \int_{-\pi}^{\pi} \frac{\left|V\left(r e^{i x}\right)\right|^{q}}{(1-r)^{1-\alpha q}} d r d x<\infty
$$

for $1<p$, where $\frac{1}{p}+\frac{1}{q}=1$, generates a bounded functional on $\Lambda_{\alpha}^{p}$, which acts by the formula

$$
l(f)=\lim _{r \rightarrow 1-0} \frac{1}{2 \pi} \int_{-\pi}^{\pi} V\left(r e^{i t}\right) f(t) d t
$$

Proof. By Lemma 3.2, the limit

$$
\lim _{r \rightarrow 1-0} \frac{1}{2 \pi} \int_{-\pi}^{\pi} V\left(r e^{i t}\right) f(t) d t
$$


exists and is equal to

$$
l(f)=\frac{V(0)}{2 \pi} \int_{-\pi}^{\pi} f(t) d t+\frac{1}{\pi} \int_{0}^{1} \int_{-\pi}^{\pi} V\left(r e^{i t}\right) \frac{\partial F\left(r e^{i t}\right)}{\partial r} d r d t .
$$

Observe that this is a bounded functional.

The lemma below is similar to the well-known result on partitions of unity; see [7. p. 45]. Nevertheless, there is an essential difference: in the result mentioned above, a cover is constructed, whereas in our lemma it is given arbitrarily beforehand.

Let $\Delta$ be an arc on the unit circle. By $\Delta^{*}$ we denote the new arc obtained by joining two arcs of the same length $|\Delta|$ to both sides of $\Delta$.

Lemma 3.3. Suppose that closed arcs $\Delta_{n}, n=1,2, \ldots$, cover the unit circle and each point $z$ with $|z|=1$ belongs to two arcs at most. Then there are nonnegative, piecewise differentiable functions $\varphi_{n}(z), n=1,2, \ldots$, satisfying the following conditions:

1) for each $n=1,2, \ldots$ we have

$$
\operatorname{supp}\left(\varphi_{n}\right) \subset \Delta_{n}^{*}
$$

2) for each point $e^{i x}$ except at most finitely many, we have

$$
\left|\frac{\partial \varphi_{k}\left(e^{i x}\right)}{\partial x}\right| \leq \frac{8}{\left|\Delta_{k}\right|} \quad k=1,2, \ldots ;
$$

3) for each point $|z|=1$ we have

$$
\sum_{n=1}^{\infty} \varphi_{n}(z)=1
$$

Proof. Without loss of generality we may assume that

$$
\left|\Delta_{1}\right| \geq\left|\Delta_{2}\right| \geq \cdots \text {. }
$$

We introduce a continuous function $\varphi_{1}(z)$ that equals 1 on the closed arc $z \in \Delta_{1}$ and is identically zero outside of $\Delta_{1}^{*}$. On $\Delta_{1}^{*} \backslash \Delta_{1}$ this function satisfies the condition

$$
\left|\frac{\partial \varphi_{1}\left(e^{i x}\right)}{\partial x}\right|=\frac{1}{\left|\Delta_{1}\right|} \text {. }
$$

Assume that the functions $\varphi_{k}(z), k=1,2, \ldots, n-1$, have already been constructed so that the following conditions are satisfied:

1) for each $k=1,2, \ldots, n-1$ we have $\operatorname{supp}\left(\varphi_{k}\right) \subset \Delta_{k}^{*}$;

2) for each $e^{i x}$ except finitely many points, we have

$$
\left|\frac{\partial \varphi_{k}\left(e^{i x}\right)}{\partial x}\right| \leq \frac{1}{\left|\Delta_{k}\right|}+\sum_{p=1}^{k-1}\left|\frac{\partial \varphi_{p}\left(e^{i x}\right)}{\partial x}\right|, \quad k=1,2, \ldots, n-1 ;
$$

3) for each point

$$
e^{i x} \in \bigcup_{k=1}^{n-1} \Delta_{k}
$$

we have

$$
\sum_{k=1}^{n-1} \varphi_{k}\left(e^{i x}\right)=1
$$

4) for each point $e^{i x}$ we have

$$
\sum_{k=1}^{n-1} \varphi_{k}\left(e^{i x}\right) \leq 1
$$


Let a point $|z|=1$ be off the arc $\Delta_{k}$. We denote by $\Delta_{k}(z)$ the arc of minimal length that contains $z$ and has nonempty intersection with the arc $\Delta_{k}$.

Now, let a new function $\psi_{n}(z)$ be defined as follows:

$$
\psi_{n}(z)=1-\sum_{k=1}^{n-1} \varphi_{k}(z)
$$

if $z \in \Delta_{n}$. For an arbitrary point $e^{i s}$ off the arc $\Delta_{n}$, we put

$$
\psi_{n}\left(e^{i s}\right)=\max \left\{c ; c+\frac{|s-t|}{\left|\Delta_{n}\right|} \leq 1-\sum_{k=1}^{n-1} \varphi_{k}\left(e^{i t}\right) \quad \text { for } \quad e^{i t} \in \Delta_{n}\left(e^{i s}\right)\right\} .
$$

Note that if a point $z,|z|=1$, is off $\Delta_{n}^{*}$, then $\psi_{n}(z)<0$. For $|z|=1$ we have

$$
\psi_{n}(z)=1-\sum_{k=1}^{n-1} \varphi_{k}(z)
$$

or

Finally, we put

$$
\left|\frac{\partial \psi_{n}\left(e^{i x}\right)}{\partial x}\right|=\frac{1}{\left|\Delta_{n}\right|}
$$

$$
\varphi_{n}(z)=\max \left\{0, \psi_{n}(z)\right\} .
$$

Obviously, $\operatorname{supp}\left(\varphi_{n}\right) \subset \Delta_{n}^{*}$. For the derivative we have the estimate

$$
\left|\frac{\partial \varphi_{n}\left(e^{i x}\right)}{\partial x}\right| \leq \frac{1}{\left|\Delta_{n}\right|}+\sum_{k=1}^{n-1}\left|\frac{\partial \varphi_{k}\left(e^{i x}\right)}{\partial x}\right| .
$$

For any $z$ with $|z|=1$ we have

$$
\left|\frac{\partial \varphi_{n}\left(e^{i x}\right)}{\partial x}\right| \leq \sum_{k=1}^{m} \frac{1}{\left|\Delta_{n_{k}}\right|}
$$

where the sum is taken over all indices $1 \leq n_{1}<n_{2}<\cdots<n_{m}=n$ such that $z \in \Delta_{n_{k}}^{*}$. We denote by $C_{j}, j=0,1, \ldots$, the set formed by the indices $k$ of the $\operatorname{arcs} \Delta_{n_{k}}$ for which

$$
2^{j}\left|\Delta_{n}\right| \leq\left|\Delta_{n_{k}}\right| \leq 2^{j+1}\left|\Delta_{n}\right| \text {. }
$$

We have

$$
\Delta_{n_{k}} \subset\left\{w ;|z-w|<2^{j+2}\left|\Delta_{n}\right|\right\}, \quad k \in C_{j} .
$$

Since $\left|\Delta_{n_{k}}\right| \geq 2^{j}\left|\Delta_{n}\right|$ and each point with $|z|=1$ belongs to at most two arcs of the family $\Delta_{n}, n=1,2, \ldots$, the set $C_{j}$ contains at most four indices. Consequently,

$$
\left|\frac{\partial \varphi_{n}\left(e^{i x}\right)}{\partial x}\right| \leq \sum_{j=0}^{\infty}\left(\sum_{k \in C_{j}} \frac{1}{\left|\Delta_{n_{k}}\right|}\right) \leq \sum_{j=0}^{\infty} \frac{4}{2^{j}\left|\Delta_{n}\right|}=\frac{8}{\left|\Delta_{n}\right|} .
$$

Lemma 3.4. Suppose $0<\alpha<1$ and $1 \leq p<\infty$. Let $\varphi(z),|z| \leq 1$, be a continuously differentiable function harmonic in the unit disk, and let $\Delta$ be an arc on the unit circle T. Suppose that

1) $0 \leq \varphi(z) \leq 1, \quad|z|<1$;

2) $\varphi(z)=0$ for each point $|z|=1$ outside of the arc $\Delta^{*}$;

3) for $|z|=1$ we have

$$
\left|\frac{\partial \varphi\left(e^{i x}\right)}{\partial x}\right| \leq \frac{4}{|\Delta|} .
$$


If $0<\sigma, 0<\epsilon$, and $0<\sigma(2 p-1)+\epsilon p<(1-\alpha) p$, then there is a constant $A$ such that for each $0<r<1$ satisfying

$$
(1-r)^{\sigma} \leq|\Delta|<1
$$

we have

$$
\|\varphi(z)-\varphi(r z)\|_{\Lambda_{\alpha}^{p}} \leq A(1-r)^{\epsilon}|\Delta| .
$$

Proof. For each $t$ we have

$$
\begin{aligned}
& \left(\frac{1}{2 \pi} \int_{-\pi}^{\pi}\left|\varphi\left(e^{i x}\right)-\varphi\left(e^{i(x+t)}\right)\right|^{p} d x\right)^{\frac{1}{p}} \\
& \quad \leq\left(\frac{1}{2 \pi} \int_{-\pi}^{\pi}\left|\varphi\left(e^{i x}\right)\right|^{p} d x\right)^{\frac{1}{p}}+\left(\frac{1}{2 \pi} \int_{-\pi}^{\pi}\left|\varphi\left(e^{i(x+t)}\right)\right|^{p} d x\right)^{\frac{1}{p}} \leq 2\left(\frac{3|\Delta|}{2 \pi}\right)^{\frac{1}{p}}
\end{aligned}
$$

If $|t|<3|\Delta|$, then, since $\operatorname{supp}\left(\varphi_{n}^{\prime}\right) \subset \Delta_{n}^{*}$, we get a better estimate:

$$
\left(\frac{1}{2 \pi} \int_{-\pi}^{\pi}\left|\varphi\left(e^{i x}\right)-\varphi\left(e^{i(x+t)}\right)\right|^{p} d x\right)^{\frac{1}{p}}=\left(\frac{1}{2 \pi} \int_{e^{i x} \in \Delta^{*}}\left|\int_{x}^{x+t} \frac{\partial \varphi\left(e^{i s}\right)}{\partial s} d s\right|^{p} d x\right)^{\frac{1}{p}} \leq \frac{8|t|}{|\Delta|^{\frac{p-1}{p}}}
$$

Consequently,

$$
\left(\frac{1}{2 \pi} \int_{-\pi}^{\pi}\left|\varphi\left(e^{i x}\right)-\varphi\left(e^{i(x+t)}\right)\right|^{p} d x\right)^{\frac{1}{p}} \leq \min \left\{2|\Delta|^{\frac{1}{p}}, \frac{8|t|}{|\Delta|^{\frac{p-1}{p}}}\right\}, \quad 0<r<1 .
$$

Since $\varphi(z),|z|<1$, is a harmonic function, for $0<r<1$ we have

$$
\frac{1}{2 \pi} \int_{-\pi}^{\pi}\left|\varphi\left(r e^{i x}\right)-\varphi\left(r e^{i(x+t)}\right)\right|^{p} d x \leq \frac{1}{2 \pi} \int_{-\pi}^{\pi}\left|\varphi\left(e^{i x}\right)-\varphi\left(e^{i(x+t)}\right)\right|^{p} d x .
$$

Consequently,

$$
\begin{aligned}
& \int_{-\pi}^{\pi} \int_{-\pi}^{\pi}\left|\left[\varphi\left(e^{i x}\right)-\varphi\left(r e^{i x}\right)\right]-\left[\varphi\left(e^{i(x+t)}\right)-\varphi\left(r e^{i(x+t)}\right)\right]\right|^{p} d x \frac{d t}{|t|^{1+\alpha p}} \\
& \quad \leq\left(\int_{|t|<\delta}+\int_{\delta<|t|<\pi}\right) \int_{-\pi}^{\pi} \mid\left[\varphi\left(e^{i x}\right)-\varphi\left(r e^{i x}\right)\right] \\
& \quad-\left.\left[\varphi\left(e^{i(x+t)}\right)-\varphi\left(r e^{i(x+t)}\right)\right]\right|^{p} d x \frac{d t}{|t|^{1+\alpha p}} \\
& \quad \leq 2 \int_{|t|<\delta}\left(\int_{-\pi}^{\pi}\left|\varphi\left(e^{i x}\right)-\varphi\left(e^{i(x+t)}\right)\right|^{p} d x\right) \frac{d t}{|t|^{1+\alpha p}}+\frac{M}{\delta^{\alpha p}} \int_{-\pi}^{\pi}\left|\varphi\left(e^{i x}\right)-\varphi\left(r e^{i x}\right)\right|^{p} d x \\
& \quad \leq A \frac{|\delta|^{(1-\alpha) p}}{|\Delta|^{p-1}}+\frac{M}{\delta^{\alpha p}} \int_{-\pi}^{\pi}\left|\varphi\left(e^{i x}\right)-\varphi\left(r e^{i x}\right)\right|^{p} d x .
\end{aligned}
$$


Also, we have

$$
\begin{aligned}
\left(\frac{1}{2 \pi}\right. & \left.\int_{-\pi}^{\pi}\left|\varphi\left(e^{i x}\right)-\varphi\left(r e^{i x}\right)\right|^{p} d x\right)^{\frac{1}{p}} \\
& \leq\left(\frac{1}{2 \pi} \int_{-\pi}^{\pi}\left|\frac{1}{2 \pi} \int_{-\pi}^{\pi} \frac{1-r^{2}}{\left|1-r e^{i t}\right|^{2}}\left(\varphi\left(e^{i x}\right)-\varphi\left(e^{i(x-t)}\right)\right) d t\right|^{p} d x\right)^{\frac{1}{p}} \\
& \leq \frac{1}{2 \pi} \int_{-\pi}^{\pi}\left(\frac{1}{2 \pi} \int_{-\pi}^{\pi} \frac{\left(1-r^{2}\right)^{p}}{\left|1-r e^{i t}\right|^{2 p}}\left|\varphi\left(e^{i x}\right)-\varphi\left(e^{i(x-t)}\right)\right|^{p} d x\right)^{\frac{1}{p}} d t \\
& \leq \frac{1}{2 \pi} \int_{-\pi}^{\pi}\left(\frac{1}{2 \pi} \int_{-\pi}^{\pi}\left|\varphi\left(e^{i x}\right)-\varphi\left(e^{i(x-t)}\right)\right|^{p} d x\right)^{\frac{1}{p}} \frac{1-r^{2}}{\left|1-r e^{i t}\right|^{2}} d t \\
& \leq \frac{M}{|\Delta|^{\frac{1}{q}}} \int_{0}^{|\Delta|} \frac{1-r^{2}}{\left|1-r e^{i t}\right|^{2}} t d t+M|\Delta|^{\frac{1}{p}} \int_{|\Delta|}^{\pi} \frac{1-r^{2}}{\left|1-r e^{i t}\right|^{2}} d t \\
& \leq M \frac{1-r}{|\Delta|^{\frac{1}{q}}} \ln \frac{|\Delta|}{1-r}, \quad 0<r<1 .
\end{aligned}
$$

Putting $\delta=(1-r)$, we get

$$
\|\varphi(z)-\varphi(r z)\|_{\Lambda_{\alpha}^{p}} \leq M \frac{(1-r)^{1-\alpha}}{|\Delta|^{\frac{1}{q}}} \ln \frac{|\Delta|}{1-r}, \quad 0<r<1 .
$$

Recalling that

$$
(1-r)^{\sigma} \leq|\Delta|
$$

where $0<\sigma(2 p-1)+\epsilon p<(1-\alpha) p$ and $0<\epsilon$, we arrive at the required inequality.

The particular case of the following result can be found in [4].

Theorem 3.2. Suppose $1 \leq p<\infty$ and $0<\alpha p<1$. Let $U(z),|z|<1$, be a harmonic function satisfying the condition

$$
|U(z)| \leq \frac{M}{(1-|z|)^{\alpha}}, \quad|z|<1
$$

for $p=1$ and the condition

$$
\int_{0}^{1} \int_{-\pi}^{\pi} \frac{\left|U\left(r e^{i x}\right)\right|^{q}}{(1-r)^{1-\alpha q}} d r d x<\infty
$$

for $p>1$, where $\frac{1}{p}+\frac{1}{q}=1$. Let $E$ be a closed subset of the unit circle, and let

$$
M_{1-\alpha p}(E)=0 .
$$

Suppose that for each $\xi \notin T \backslash E$ we have

$$
\liminf _{r \rightarrow 1-0}\left(\max \left\{U(z) ;|z|=r,|z-\xi| \leq A(1-r)^{\sigma}\right\}\right) \leq 0,
$$

where $0<\sigma(2 p-1)<(1-\alpha) p$. Then $U(z) \leq 0,|z|<1$.

Proof. Let $f(z), z \in T$, be a nonnegative function with $\operatorname{supp}(f) \subset I$, where $I$ is an arc in $T \backslash E$. Let $0<r_{0}<1$ be an arbitrary number. Then for each $\epsilon>0$ and each point $\xi \in I$, there is a number $r(\xi)$ satisfying the condition $r_{0}<r(\xi)<1$ and such that $U(z) \leq \epsilon$ for

$$
z \in\left\{w ; w \in \Delta_{\sigma}(\xi), r(\xi)<|w|<1\right\} .
$$

Without loss of generality we may assume that the arcs

$$
C(\xi)=\left\{\frac{z}{|z|} ;|z|=r(\xi), z \in \Delta_{\sigma}(\xi)\right\}
$$


are subsets of $T \backslash E$. Observe that these arcs cover $I$. By the Ahlfors lemma (see [8, p. 246]), there are intervals

$$
C\left(\xi_{n}\right), \quad n=1,2, \ldots,
$$

that also cover the arc $I$ and have multiplicity less than 2. For this family of intervals, we consider the functions

$$
\varphi_{n}(z), \quad|z|=1, \quad n=1,2, \ldots,
$$

constructed as in Lemma 3.3.

Let $f(z), z \in T$, be a continuously differentiable function with $\operatorname{supp}(f) \subset I$, and let $g_{n}(z),|z|<1$, denote the harmonic function for which

$$
g_{n}(z)=\varphi_{n}(z) f(z), \quad z \in T \text {. }
$$

We put

$$
\psi_{n}(z)=g_{n}\left(r\left(\xi_{n}\right) z\right), \quad|z| \leq 1
$$

Let $l \in\left(\Lambda_{\alpha}^{p}\right)^{*}$ be the functional generated by the harmonic function $U(z)$. We have

$$
l(f)=\sum_{n=1}^{\infty} l\left(f \varphi_{n}\right)=\sum_{n=1}^{\infty} l\left(f \varphi_{n}-\psi_{n}\right)+\sum_{n=1}^{\infty} l\left(\psi_{n}\right) .
$$

Observe that

$$
\begin{aligned}
l\left(\psi_{n}\right) & =\lim _{r \rightarrow 1-0} \frac{1}{2 \pi} \int_{-\pi}^{\pi} U\left(r e^{i t}\right) g_{n}\left(r\left(\xi_{n}\right) e^{i t}\right) d t \\
& =\frac{1}{2 \pi} \int_{-\pi}^{\pi} U\left(r\left(\xi_{n}\right) e^{i t}\right) g_{n}\left(e^{i t}\right) d t=\frac{1}{2 \pi} \int_{-\pi}^{\pi} U\left(r\left(\xi_{n}\right) e^{i t}\right) \varphi_{n}\left(e^{i t}\right) f\left(e^{i t}\right) d t .
\end{aligned}
$$

Since

$$
U\left(r\left(\xi_{n}\right) e^{i t}\right) \leq \epsilon
$$

whenever $e^{i t} \in \operatorname{supp}\left(\varphi_{n}\right) \subset C\left(\xi_{n}\right)$, we have

$$
\begin{aligned}
& \sum_{n=1}^{\infty} \frac{1}{2 \pi} \int_{-\pi}^{\pi} U\left(r\left(\xi_{n}\right) e^{i t}\right) \varphi_{n}\left(e^{i t}\right) f\left(e^{i t}\right) d t \\
& \quad \leq \epsilon \sum_{n=1}^{\infty} \frac{1}{2 \pi} \int_{-\pi}^{\pi} \varphi_{n}\left(e^{i t}\right) f\left(e^{i t}\right) d t \leq \epsilon \frac{1}{2 \pi} \int_{-\pi}^{\pi} f\left(e^{i t}\right) d t
\end{aligned}
$$

Now, choosing the number $r_{0}<1$ close to 1 and using Lemma 2, we can write

$$
\left|l\left(f \varphi_{n}-\psi_{n}\right)\right| \leq M\left\|f \varphi_{n}-\psi_{n}\right\|_{\Lambda_{\alpha}^{p}} \leq \epsilon\left|C\left(\xi_{n}\right)\right| .
$$

This proves that $l(f) \leq 0$ for any function with $\operatorname{supp}(f) \subset T \backslash E$. It remains to apply Lemma 1.

\section{§4. The CASE Where the Lengths OF THE ARCS DO NOT GO TO ZERO}

If no boundary points are omitted, then the following is true.

Theorem 4.1. Let $U(z)$ be a harmonic function defined on the unit disk $D$ and satisfying the condition

$$
\int_{0}^{1} \ln ^{+} \ln ^{+}\left(\max _{x}\left|U\left(r e^{i x}\right)\right|\right) d r<\infty .
$$

If there is a number $\epsilon>0$ such that

$$
\liminf _{r \rightarrow 1-0}\left(\max \left\{U\left(r e^{i y}\right) ;|x-y| \leq \epsilon\right\}\right) \leq 0
$$

for any boundary point $e^{i x} \in \partial D$, then $U(z) \leq 0$ everywhere on the unit disk $D$. 
Proof. We write the harmonic function $U(z),|z|<1$, in the form

$$
U(z)=\sum_{n=-\infty}^{\infty} a_{n} r^{|n|} e^{i n x}, \quad z=r e^{i x}
$$

and denote

$$
M(r)=\ln ^{+}\left(\max _{x}\left|U\left(r e^{i x}\right)\right|\right) .
$$

The function $M(r)$ is monotone increasing for $0<r_{0}<r<1$. The coefficients $a_{n}, n=1$, $2, \ldots$, admit the estimate

$$
\left|a_{n}\right| \leq r^{-|n|} \max _{|x| \leq \pi}\left|U\left(r e^{i x}\right)\right|, \quad 0<r<1 .
$$

Consequently,

$$
\left|a_{n}\right| \leq \exp (M(r)+2|n|(1-r)), \quad \frac{1}{2} \leq r<1 .
$$

For each $n=1,2, \ldots$, we choose $0<r_{n}<1$ such that

$$
M\left(r_{n}\right)=2|n|\left(1-r_{n}\right) .
$$

Note that for large indices we have $r_{n}<r_{n+1}$, and $\lim _{n \rightarrow \infty} r_{n}=1$.

Putting $t_{n}=M\left(r_{n}\right)$, we get

$$
\begin{aligned}
\infty & >\int_{0}^{1} \ln M(r) d r=\int_{1}^{\infty} m(\{r ; M(r)>t\}) \frac{d t}{t} \\
& \geq \sum_{n=1}^{\infty}\left(1-r_{n}\right) \frac{t_{n+1}-t_{n}}{t_{n}}=\sum_{n=1}^{\infty}\left(1-r_{n}\right) \frac{M\left(r_{n+1}\right)-M\left(r_{n}\right)}{M\left(r_{n}\right)} \\
& =\sum_{n=1}^{\infty}\left(-\left(r_{n+1}-r_{n}\right)+\frac{1-r_{n+1}}{n}\right)>\sum_{n=2}^{\infty} \frac{M\left(r_{n}\right)}{2 n^{2}}-1 .
\end{aligned}
$$

Also, we have

$$
\left|a_{n}\right|+\left|a_{-n}\right| \leq \exp \left\{2 M\left(r_{n}\right)\right\}, \quad n=0,1, \ldots .
$$

Let $0<\delta<\epsilon$ be a fixed number. By Theorem 2.2, there is a nonnegative function $\varphi_{\delta}(x)$ supported on the interval $(-\delta, \delta)$ and satisfying

$$
\frac{1}{2 \pi} \int_{-\delta}^{\delta} \varphi_{\delta}(x) d x=1
$$

and

$$
\left|c_{n}\right| \leq \exp \left\{-2 M\left(r_{n}\right)-\sqrt{n}\right\}, \quad n=1,2, \ldots,
$$

where the $c_{n}$ are the Fourier coefficients of the function $\varphi_{\delta}(x)$.

In particular, by (3) we have

$$
\sum_{n=-\infty}^{\infty}\left|a_{n}\right|\left|c_{n}\right|<\infty
$$

Consider the function

$$
U_{\delta}(z)=\frac{1}{2 \pi} \int_{-\pi}^{\pi} U\left(z e^{-i x}\right) \varphi_{\delta}(x) d x .
$$

This function is harmonic and admits a continuous extension up to the boundary of $D$. By the assumptions of the theorem, for any $w \in \partial D$ there exists a sequence $z_{n}$, $n=1,2, \ldots$, in $D$ that tends to $w$ and is such that

$$
\lim _{D \ni z_{n} \rightarrow w} U_{\delta}\left(z_{n}\right) \leq 0 .
$$


Since $U_{\delta}(z)$ is continuous up to the boundary, we have $U_{\delta}(w) \leq 0$. By the maximum principle, $U_{\delta}(z) \leq 0$ for $z \in D$. Hence, $U(z)=\lim _{\delta \rightarrow 0} U_{\delta}(z) \leq 0$.

Theorem 4.2. There is a number $\delta>0$ and a function analytic in the unit disk such that

and

$$
|f(z)| \leq \exp \left\{\exp \left\{\frac{C}{(1-|z|)}\right\}\right\}, \quad|z|<1,
$$

$$
\liminf _{r \rightarrow 1-0}\left(\max _{|t| \leq \delta}\left|f\left(r e^{i(y+t)}\right)\right|\right) \leq 1
$$

for every $e^{i y} \in \partial D$, but $f(z)$ is unbounded.

Proof. Consider the function

$$
w(z)=z^{i}\left(1-\frac{b}{z}\right)=\left(1-\frac{b}{z}\right) e^{-\arg (z)+i \ln |z|},
$$

defined on the strip

$$
E(a)=\left\{z=x+i y ; a<x,|y| \leq \frac{\pi}{2}\right\},
$$

where $16 \pi<4 b<a$. For each $a<x<\infty$ we have

$$
\arg (w(x))=\arg \left(w\left(x e^{2 \pi}\right)\right) .
$$

We denote

$$
\Omega(a)=\{w(z) ; z \in E(a)\} .
$$

For each $z \in \Omega(a)$ we have $x \leq|z| \leq x+\frac{\pi}{2}$. Therefore,

$$
\frac{b^{2}}{|z|^{2}}+\frac{\pi}{x} \leq \frac{b^{2}}{x^{2}}+\frac{\pi}{x}<\frac{b}{2 x}<\frac{2 b x}{\left(x+\frac{\pi}{2}\right)^{2}} \leq \frac{2 b x}{|z|^{2}} .
$$

It follows that

$$
\begin{aligned}
\left|1-\frac{b}{z}\right|^{2} & <1-\frac{\pi}{x}<\left(1-\frac{\pi}{2 x}\right)^{2} \leq\left(1-\frac{|y|}{x}\right)^{2} \\
& =\left(1-\tan (|\arg (z)|)^{2} \leq(1-|\arg (z)|)^{2} \leq e^{-2|\arg (z)|} .\right.
\end{aligned}
$$

Consequently, $|w(z)|<1$ for $z \in E(a)$. In other words, $\Omega(a) \subset D$.

For $z \in E(a)$ we have

$$
\begin{aligned}
w(x+i y) & =\left(1-\frac{b}{z}\right) e^{-\arg (z)+i \ln |z|} \\
& =\left(1-\frac{b}{x}+O\left(\frac{1}{x^{2}}\right)\right)\left(1-\frac{y}{x}+O\left(\frac{1}{x^{2}}\right)\right) e^{i \ln x} \exp \left\{\frac{i}{2} \ln \left(1+\frac{y^{2}}{x^{2}}\right)\right\} \\
& =\left(1-\frac{b+y}{x}\right) e^{i \ln x}+O\left(\frac{1}{x^{2}}\right), \quad x \rightarrow \infty .
\end{aligned}
$$

The spiral

$$
S=\{w(x) ; a<x<\infty\}
$$

goes to the boundary of the unit disk $D$. As $x \rightarrow+\infty$, we have

$$
\begin{aligned}
& \inf \{|w(x)-w| ; w \in \partial \Omega(a)\} \\
& \quad \geq\left|w(x)-w\left(x-\frac{\pi}{2} i\right)\right|+O\left(\frac{1}{x^{2}}\right) \geq \frac{\pi}{2 x}+O\left(\frac{1}{x^{2}}\right) .
\end{aligned}
$$

Observe that the inequality

$$
\left|w\left(x-\frac{\pi i}{2}\right)\right|<\left|w\left(x e^{2 \pi}+\frac{\pi i}{2}\right)\right|
$$




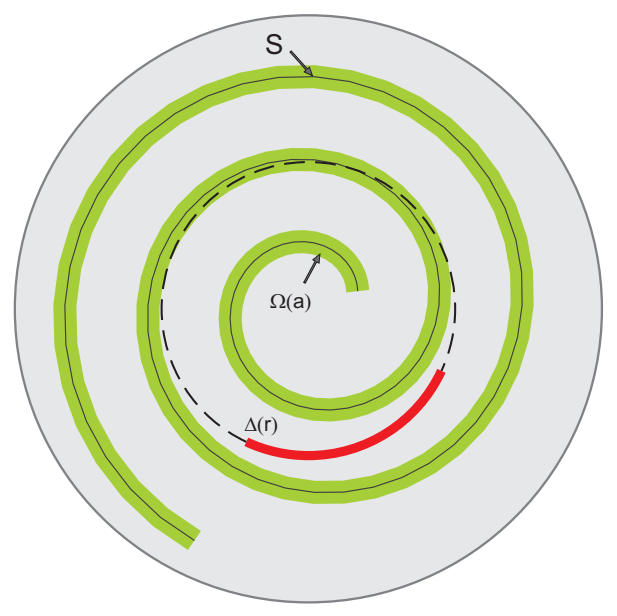

is fulfilled if

$$
1-\frac{2 b-\pi}{2 x}<1-\frac{2 b+\pi}{2 x e^{2 \pi}}
$$

because

$$
\frac{\pi\left(e^{2 \pi}+1\right)}{2\left(e^{2 \pi}-1\right)}<4 \pi<b
$$

So, the linear interval with the endpoints $w\left(x e^{2 \pi}+\frac{\pi i}{2}\right)$ and $w\left(x-\frac{\pi i}{2}\right)$ lies off the domain $\Omega(a)$, and we have

$$
\left|w\left(x e^{2 \pi}+\frac{\pi i}{2}\right)\right|-\left|w\left(x-\frac{\pi i}{2}\right)\right|=\frac{1}{x}\left(\frac{2 b-\pi}{2}-\frac{2 b+\pi}{2 e^{2 \pi}}\right)+O\left(\frac{1}{x^{2}}\right) .
$$

It follows that the inequalities

$$
\begin{aligned}
& \frac{1}{3}\left(\left|w\left(x e^{2 \pi}+\frac{\pi i}{2}\right)\right|+2\left|w\left(x-\frac{\pi i}{2}\right)\right|\right) \\
& \quad<r<\frac{1}{3}\left(2\left|w\left(x e^{2 \pi}+\frac{\pi i}{2}\right)\right|+\left|w\left(x-\frac{\pi i}{2}\right)\right|\right)
\end{aligned}
$$

are valid for $x \in F(r)$, where

$$
F(r)=\left[\frac{2 b+\pi}{3 e^{2 \pi}(1-r)}+\frac{2 b-\pi}{6(1-r)}, \frac{2 b+\pi}{6 e^{2 \pi}(1-r)}+\frac{2 b-\pi}{3(1-r)}\right] .
$$

We denote

$$
\Delta(r)=\left\{r e^{i \ln x} ; x \in F(r)\right\} .
$$

The length of this arc is equal to

$$
|\Delta(r)|=\ln \frac{2 b+\pi+2 e^{2 \pi}(2 b-\pi)}{4 b+2 \pi+e^{2 \pi}(2 b-\pi)}=\delta>0 .
$$

For each $x \in F(r)$, we have

$$
\inf \left\{\left|r e^{i \ln x}-w\right| ; w \in \partial \Omega(a)\right\} \geq \frac{1}{3 x}\left(\frac{2 b-\pi}{2}-\frac{2 b+\pi}{2 e^{2 \pi}}\right)+O\left(\frac{1}{x^{2}}\right) .
$$

We note that

$$
w(z): E(a) \rightarrow \Omega(a)
$$


is a conformal mapping. Indeed, we have

$$
\begin{aligned}
& \left|w\left(x+i \frac{\pi}{2}\right)\right|^{2}=\frac{4(x-b)^{2}+\pi^{2}}{4 x^{2}+\pi^{2}} \exp \left\{-2 \arctan \left(\frac{\pi}{2 x}\right)\right\}, \\
& \left|w\left(x-i \frac{\pi}{2}\right)\right|^{2}=\frac{4(x-b)^{2}+\pi^{2}}{4 x^{2}+\pi^{2}} \exp \left\{2 \arctan \left(\frac{\pi}{2 x}\right)\right\},
\end{aligned}
$$

and

$$
|w(a+i y)|^{2}=\frac{(a-b)^{2}+y^{2}}{a^{2}+y^{2}} \exp \left\{-2 \arctan \left(\frac{y}{a}\right)\right\} .
$$

Since

$$
\begin{aligned}
& x^{2} \frac{d}{d x} \ln \left|w\left(x+i \frac{\pi}{2}\right)\right|^{2}=2 b+\frac{\pi}{2}+o(1), \\
& x^{2} \frac{d}{d x} \ln \left|w\left(x-i \frac{\pi}{2}\right)\right|^{2}=2 b-\frac{\pi}{2}+o(1),
\end{aligned}
$$

and

$$
\frac{d}{d y} \ln |w(a+i y)|^{2}=-\frac{2 a}{a^{2}+y^{2}}\left(1-\frac{y b(2 a-b)}{a\left(y^{2}+(a-b)^{2}\right)}\right),
$$

for sufficiently large $a$ the function $w(z), z \in \partial E(a)$, realizes a one-to-one mapping onto $\partial \Omega(a)$.

Let $\xi=\xi(w), w \in \Omega(a)$, be the inverse to the function $w=w(\xi), \xi \in E(a)$.

On the domain $z \in D \backslash \Omega(a)$, we consider the analytic function

$$
f_{-}(z)=\frac{1}{2 \pi i} \int_{\gamma(a)} \frac{\exp \{\exp \{\xi(w)\}\}}{(w-z) \xi^{2}(w)} d w
$$

where $\gamma(a)=\partial \Omega(a) \cap\{w ;|w|<1\}$ and the direction on $\gamma(a)$ is positive with respect to the domain $\Omega(a)$.

By the same formula (5), we define a function $f_{+}(z)$ for $z \in \Omega(a)$.

For $z \in D \backslash \Omega(\overline{(} a)$ we have

$$
\begin{aligned}
f_{-}(z)= & \frac{1}{2 \pi i} \int_{\partial E(a)} \frac{w^{\prime}(\xi) \exp \{\exp \{\xi\}\}}{(w(\xi)-z) \xi^{2}} d \xi \\
= & \frac{1}{2 \pi i} \int_{\partial E(a)} \frac{\xi^{i} \exp \{\exp \{\xi\}\}}{(w(\xi)-z)}\left(\frac{i}{\xi^{3}}-\frac{b(i-1)}{\xi^{4}}\right) d \xi \\
= & -\frac{1}{2 \pi i} \int_{+\infty}^{a} \frac{(x-i \pi / 2)^{i}}{(w(x-i \pi / 2)-z)}\left(\frac{i}{(x-i \pi / 2)^{3}}-\frac{b(i-1)}{(x-i \pi / 2)^{4}}\right) \exp \{-i \exp \{x\}\} d x \\
& +\frac{1}{2 \pi i} \int_{a}^{+\infty} \frac{(x+i \pi / 2)^{i}}{(w(x+i \pi / 2)-z)}\left(\frac{i}{(x+i \pi / 2)^{3}}-\frac{b(i-1)}{(x+i \pi / 2)^{4}}\right) \exp \{-i \exp \{x\}\} d x \\
& +\frac{1}{2 \pi} \int_{-\pi / 2}^{\pi / 2} \frac{(a+i y)^{i}}{(w(a+i y)-z)}\left(\frac{i}{\pi \alpha(a+i y)^{3}}-\frac{b(i-1)}{(a+i y)^{4}}\right) \exp \{\exp \{a+i y\}\} d y .
\end{aligned}
$$

Observe that the function $f_{-}(z)$ takes the same value for each $a$ if $z \in D \backslash \overline{\Omega(a)}$. Consequently, the function $f_{-}(z)$ admits analytic continuation to the unit disk, which will be denoted by $f(z),|z|<1$.

Now we estimate the function $f(z)$. For an arbitrary point $z,|z|<1$, we take $a<c<$ $+\infty$ so that $|w(c)|=\frac{|z|+1}{2}$. Since $|w(c)|=1-\frac{b}{c}+O\left(\frac{1}{c^{2}}\right)$, we have

$$
c=\frac{2 b}{1-|z|}+O(1) \text {. }
$$


Consequently,

$$
\begin{aligned}
\left|f_{-}(z)\right| & \leq A \int_{c}^{+\infty} \frac{d x}{(1-|z|)\left(x^{3}+1\right)} d x+\frac{B}{1-|z|} \exp \{\exp \{c\}\} \\
& \leq \frac{C}{1-|z|} \exp \left\{\exp \left\{\frac{C}{1-|z|}\right\}\right\} .
\end{aligned}
$$

Note that the function $f_{-}(z)$ is bounded on the arcs $\Delta(r)$ and the function $f_{+}(z)$ is bounded on the spiral $S$.

For each boundary point $e^{i x}$, there are infinitely many numbers $0<r_{n}<1$ such that the points $r_{n} e^{i x}$ are the centers of the $\operatorname{arcs} \Delta\left(r_{n}\right)$. So, the condition of the theorem is satisfied.

Now we show that the function $f_{-}(z),|z|<1$, is not bounded. Indeed, otherwise for any $w \in \partial \Omega(a) \cap S$ we have

$$
\lim _{S \cap \Omega(a) \ni z \rightarrow w} f_{-}(z)-\lim _{S \backslash \Omega(a) \ni z \rightarrow w} f_{+}(z)=\frac{\exp \{\exp \{a\}\}}{a^{2}} .
$$

If the function $f_{-}(z),|z|<1$, is bounded, then the function $f_{+}(w)$ cannot be bounded on the spiral $S$, a contradiction.

\section{REFERENCES}

[1] S. Mandelbrojt, Séries de Dirichlet. Principes et méthodes, Monogr. Internat. Math. Modernes, vol. 11, Gauthier-Villars, Paris, 1969. MR0259079 (41:3721)

[2] L. Carleson, Selected problems on exceptional sets, Van Nostrand Math. Stud., No. 13, Van Nostrand Co., Inc., Princeton, NJ, 1967. MR0225986 (37:1576)

[3] E. M. Stein, Singular integrals and differentiability properties of functions, Princeton Math. Ser., No. 30, Princeton Univ. Press, Princeton, NJ, 1970. MR0290095 (44:7280)

[4] A. Vagarshakyan, On the maximum principle, Izv. Akad. Nauk Armenii Mat. 26 (1991), no. 4, 300308 (1992); English transl., Contemp. Math. Anal. 26 (1991), no. 4, 21-28. MR.1231851 (94h:30031)

[5] _ Boundary properties of certain classes of harmonic functions, Izv. Akad. Nauk Armyan. SSR Ser. Mat. 10 (1975), no. 1, 54-60. (Russian) MR0382681(52:3563)

[6] Henrik Shahgholian and Ashot Vagharshakyan, On Phragmen Lindelöf principle, Complex Variables Theory Appl. 46 (2001), no. 4, 295-305. MR1873963 (2002j:30032)

[7] L. Hörmander, The analysis of linear partial differential operators. I, Grundlehren Math. Wiss., Bd. 256, Springer-Verlag, Berlin, 1983. MR0717035(85g:35002a)

[8] N. Landkof, Foundations of modern potential theory, "Nauka", Moscow, 1966; English transl., Grundlehren Math. Wiss., Bd. 180, Springer-Verlag, New York-Heidelberg, 1972. MR0214795 (35:5644) MR0350027(50:2520)

Institute of Mathematics, Armenian National Academy of Sciences, Bagramian 24-B, 375019, Yerevan, Armenia

E-mail address: vagharshakyan@yahoo.com

Received 5/MAR/2007

Originally published in English 